\title{
Recent Results on Neutrino Oscillations (and Reactor Neutrino Spectra) from Daya Bay
}

\author{
Jim Napolitano, for the Daya Bay Collaboration*† \\ Temple University, Philadelphia PA, USA \\ E-mail: napolj@temple.edu
}

\begin{abstract}
We presented recent results from the Daya Bay neutrino experiment, including a precision measurement of $\theta_{13}$, a measurement of $\theta_{13}$ using neutron capture on hydrogen, a search for sterile neutrinos, and preliminary results on the absolute flux and spectrum shape of neutrinos from the Daya Bay nuclear power reactors.
\end{abstract}

XIIth International Conference on Heavy Quarks \& Leptons 2014,

25-29 August 2014

Schloss Waldthausen, Mainz, Germany

\footnotetext{
*Speaker.

${ }^{\dagger}$ The author is grateful to the organizers and also to the Daya Bay Collaboration for this opportunity.
} 


\section{Introduction}

The Daya Bay Reactor Neutrino Experiment $[1,2,3,4,5,6,7]$ measures $\bar{v}_{e}$ disappearance using the nuclear reactors at the Daya Bay Nuclear Power Plant as neutrino sources. This $17 \mathrm{GW}$ (thermal) power plant has six reactor cores, in pairs at two separated locations on site. The antineutrino detectors were built as functionally identical pairs, yielding a very high level of performance consistency between all detectors.

One detector near one of the two reactor locations was designed to be paired with a detector located at a far site, whose distance was chosen to optimize sensitivity to $1 \leftrightarrow 3$ oscillations. In this way, we could observe disappearance through a deficit relative to $1 / r^{2}$ dependence, removing the necessity of precisely predicting the reactor flux. The experiment has been taking data since December 2011 with six active antineutrino detectors, three of which are mounted at the far site, and since August 2012 with all eight detectors.

The phenomenology of neutrino oscillations is well understood [8] and the notation is now rather standard. For an experiment such as Daya Bay, where we optimize sensitivity to mixing between the first and third generations, it is convenient to write the disappearance probability as

$$
\begin{aligned}
P\left(\bar{v}_{e} \rightarrow \bar{v}_{e}\right)= & 1-\cos ^{4} \theta_{13} \sin ^{2} 2 \theta_{12} \sin ^{2} \Delta_{21} \\
& -\sin ^{2} 2 \theta_{13}\left(\cos ^{2} \theta_{12} \sin ^{2} \Delta_{31}+\sin ^{2} \theta_{12} \sin ^{2} \Delta_{32}\right)
\end{aligned}
$$

where

$$
\Delta_{j i} \equiv 1.267 \Delta m_{j i}^{2}\left(\mathrm{eV}^{2}\right)[L(\mathrm{~m}) / E(\mathrm{MeV})]
$$

and $\Delta m_{j i}^{2}=m_{j}^{2}-m_{i}^{2}$ is the difference in the squares of the masses for generations $i$ and $j$. It is now well known that $\Delta m_{21}^{2} \ll \Delta m_{32}^{2}$, so optimizing for $1 \leftrightarrow 3$ oscillations means that $\Delta_{31}$ is order unity, while $\Delta_{21} \ll 1$. Also, since $\Delta m_{31}^{2}=\Delta m_{21}^{2}+\Delta m_{32}^{2} \approx \Delta m_{32}^{2}$, we define a fit parameter $\Delta m_{e e}^{2}$ through

$$
\sin ^{2} \Delta_{e e} \equiv \cos ^{2} \theta_{12} \sin ^{2} \Delta_{31}+\sin ^{2} \theta_{12} \sin ^{2} \Delta_{32}
$$

in which case $\Delta m_{e e}^{2}$ is very close to $\Delta m_{31}^{2}$ and $\Delta m_{32}^{2}$.

The experiment has performed very well, with all eight 20 -ton (fiducial) detectors running with nearly identical characteristics, and the overburden and other shielding providing an exceptionally high level of background rejection. As usual for reactor neutrino experiments, we use the inverse beta decay reaction $\bar{v}_{e}+p \rightarrow e^{+}+n$ to detect the $\bar{v}_{e}$, where the target protons are an intrinsic component of the liquid scintillator detector. The scintillator is loaded with gadolinium, which has a very high neutron capture cross section, leading to $\sim 8 \mathrm{MeV}$ of photons. The $n+G d$ capture signal is delayed by tens of $\mu \mathrm{sec}$ as the neutron thermalizes from $n p$ collisions in the scintillator.

Figure 1 summarizes the detector performance. The primary signal window encompasses a well separated region that clearly shows neutron capture events on gadolinium, based on the $8 \mathrm{MeV}$ delayed energy. Selecting this region and projecting out the prompt energy spectrum, gives the histogram on the right, for a pair of detectors in one of the near halls. Various background sources are highlighted in the inset, dominated by accidental coincidences at low energy, and ${ }^{9} \mathrm{Li}$ spallation events up to high energy. The scatterplot also shows an inverse beta decay signal from $n p$ capture, and also shows the very small contribution from fast neutron backgrounds that extend up to high prompt energy. 

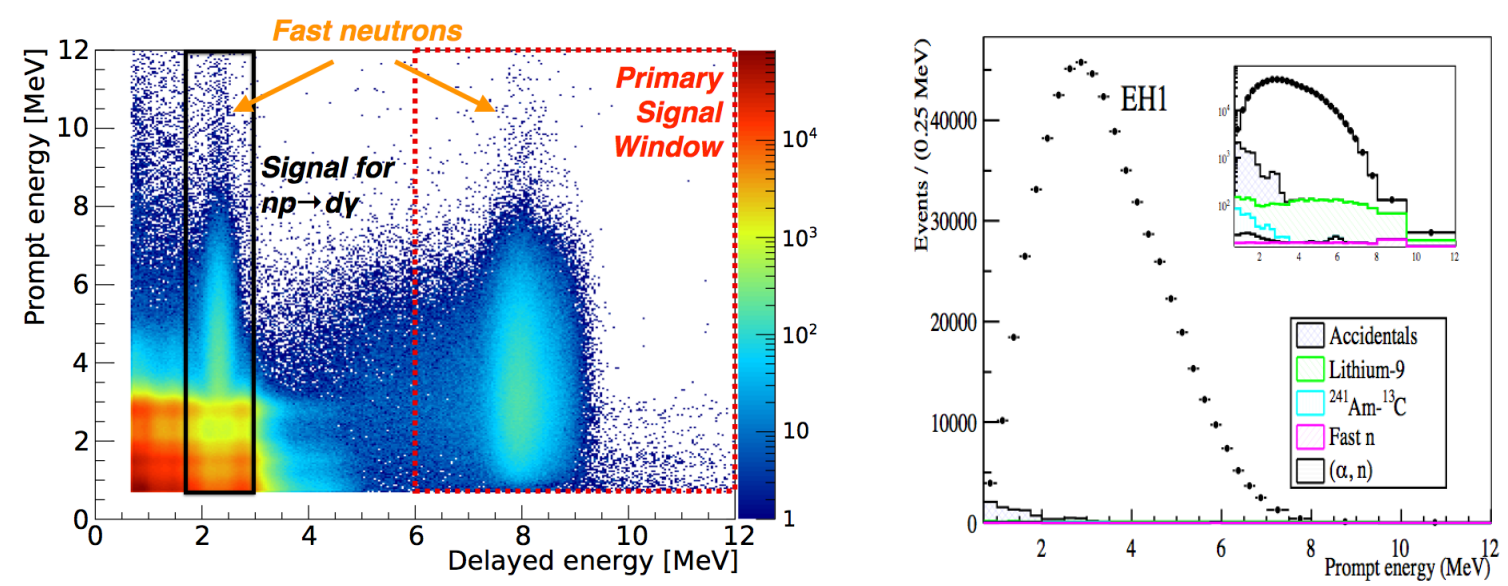

Figure 1: Performance of the Daya Bay detectors. On the left is a scatterplot of "prompt" versus "delayed" energy, showing a very clear separation of the delayed neutron capture signal on gadolinium. The right side histograms the prompt energy for events in the signal region. More detail is given in the text.

This talk covers updated and new results from our experiment in four areas. These are precision results on $\theta_{13}$ oscillations $[9,10,11]$, a cross check on $\theta_{13}$ using neutron capture on protons [12], a search for sterile neutrinos [13], and measurements of the reactor spectrum flux and shape. Some of these results have appeared in print since this talk was given, but all results were presented at the XXVI International Conference on Neutrino Physics and Astrophysics (Neutrino 2014) and the 37th International Conference on High Energy Physics (ICHEP 2014).

\section{Precision results $\bar{v}_{e}$ disappearance}

The primary goal of the Daya Bay Reactor Neutrino Experiment was to measure, or put limits on, $\sin ^{2} 2 \theta_{13}$. There was considerable speculation at the time when the current generation of reactor experiments were being designed, that $\theta_{13}$ might be very small, because the best existing limit [14] put the value well below the known values of $\sin ^{2} 2 \theta_{12}$ and $\sin ^{2} 2 \theta_{23}$. Consequently, our approach was to build an experiment with very high sensitivity, namely combined systematic and statistical uncertainty below $1 \%$.

We have indeed achieved this $1 \%$ sensitivity to $\bar{v}_{e}$ disappearance, but of course we now know that the size of the signal is much larger. Consequently, this sensitivity contributes to an especially precise value for $\sin ^{2} 2 \theta_{13}$. Our previous publications $[9,10,11]$ present results on data taken in the initial configuration of six antineutrino detectors. In this talk, we show updated results for 621 days of data, including all data with six detectors and the first portion of our running with eight detectors.

These updated results are shown in Figure 2. A fit is performed to the individual prompt energy spectra, taking into account the distribution and distances of the various reactor cores, as well as the individual detector calibrations. The absolute rate, integrated over all energies, is mainly sensitive to $\sin ^{2} 2 \theta_{13}$, while the spectrum shapes at different distances is mainly determined by $\Delta m_{e e}^{2}$, as defined in Equations 1.3 and 1.2. We find

$$
\sin ^{2} 2 \theta_{13}=0.084 \pm 0.005 \quad \text { and } \quad\left|\Delta m_{e e}^{2}\right|=2.44_{-0.11}^{+0.10} \times 10^{-3} \mathrm{eV}^{2}
$$



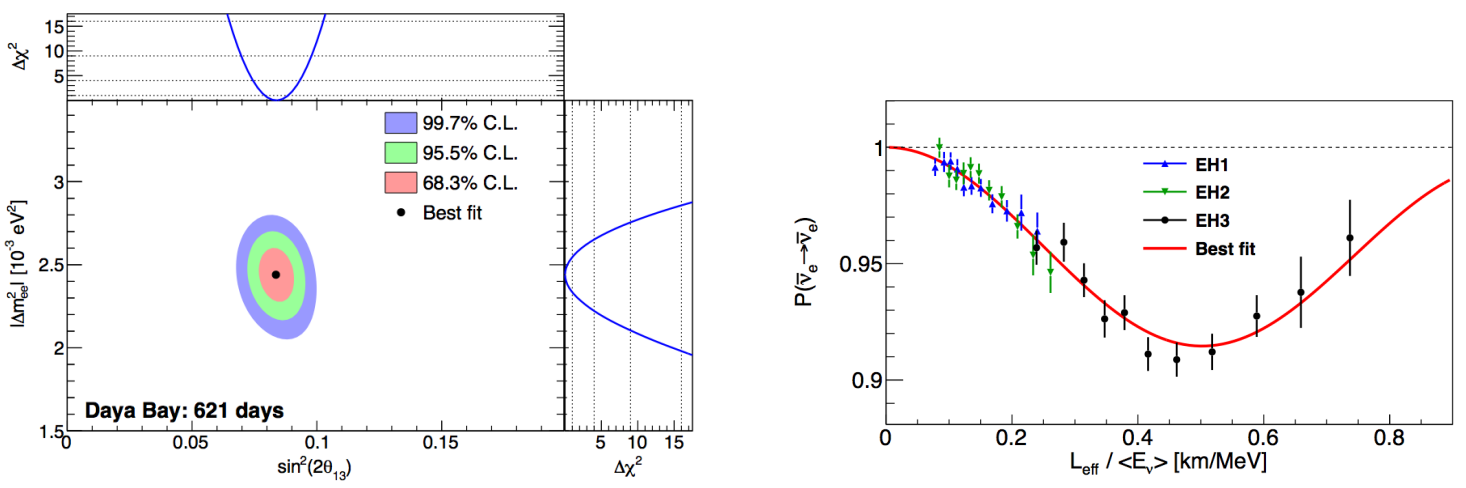

Figure 2: Most recent results from Daya Bay on $\bar{v}_{e}$ disappearance, including a fit to both the rate dependence on distance and the spectral shape distortion. See text for details.

The uncertainties are almost entirely due to statistical errors, with detector calibrations and reactor spectrum shape being the largest contributions to systematic error.

The figure on the right is a demonstration of the how well this fit reproduces Equation 1.1, using one approach to forming an effective distance $L_{\mathrm{eff}}$. The fit function shape is dominated by the third term on the right of Equation 1.1, but the presence of the second term is noticeable as well. Note that since we are using the "near" detectors to fix the absolute reactor flux, the red curve is constrained so that $P\left(\bar{v}_{e} \rightarrow \bar{v}_{e}\right)=1$ for $L_{\text {eff }}=0$.

Our experiment is approved to run through 2017, at which time our statistical and systematic uncertainties will be comparable to each other.

\section{Independent measurement of $\theta_{13}$ using $n H$ capture}

Figure 1 clearly indicates the presence of a delayed $n H$ capture signal, where a $2.2 \mathrm{MeV}$ gamma ray is emitted in $n p \rightarrow d \gamma$. Although this cross section is orders of magnitude smaller than neutron capture on gadolinium, the proton density is many orders of magnitude larger. Therefore, we can use $n p$ capture to measure inverse beta decay, leading us to cross check our result using the primary signal.

The difficulty is background. It is clear from Figure 1 that below $\sim 4 \mathrm{MeV}$ in prompt energy, the $n H$ capture signal overlaps strongly with accidental coincidence events. In order to see the "turnover" in the neutrino oscillation signal at low energies, we need to recover that portion of the spectrum. We do this by measuring the accidental coincidence spectrum using delayed events far outside the time window for neutron capture, and testing that procedure by studying various distributions after subtraction.

The left panel of Figure 3 shows the distribution in linear distance between the inverse beta decay candidate signal and the $n p$ capture signal, before and after background subtraction. (Note that our antineutrino detectors are right cylinders $5 \mathrm{~m}$ in diameter and $5 \mathrm{~m}$ high.) This and other distributions give us confidence in the background subtraction procedure.

Figure 3 also shows the result of the same fitting procedure that we use for the primary signal, once again showing $\bar{v}_{e}$ disappearance with a large amplitude, consistent with the spectral distortion. 

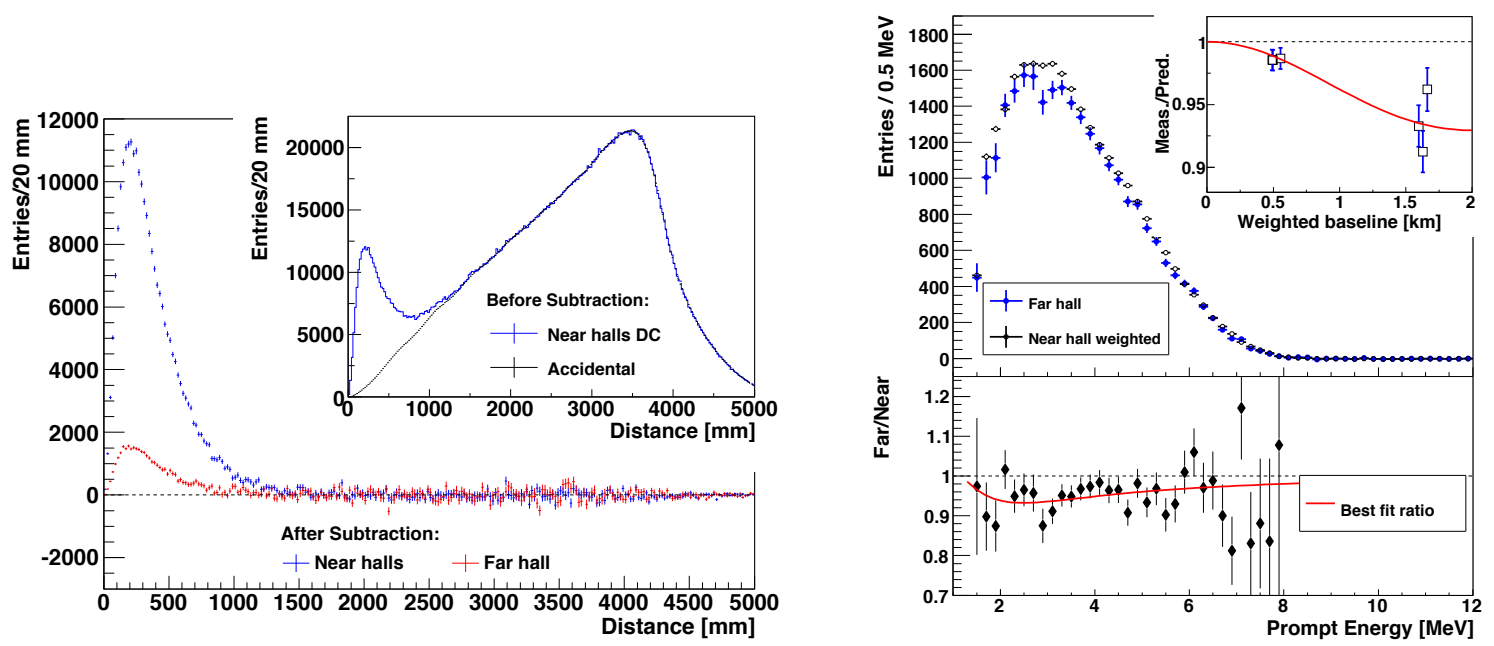

Figure 3: Measurement of $\bar{v}_{e}$ disappearance using delayed signal from $n p \rightarrow d \gamma$. The figures are taken from our recent publication [12]. The figure on the left demonstrates our background subtraction technique, aimed mainly at low prompt energy events. The right panel demonstrates our fit for the rate deficit, including its distortion on the neutrino spectrum shape.

From a rate-only analysis on 217 days of data, we find $\sin ^{2} 2 \theta_{13}=0.083 \pm 0.018$, completely consistent with our more precise result from $n G d$ capture.

\section{A search for sterile neutrinos}

Various anomalies in neutrino measurements have led some to speculate on the existence of a fourth "sterile" neutrino, with which the other generations can oscillate. One example is the so-called "Reactor Neutrino Anomaly" [15] which suggests that a systematic over-prediction of reactor $\bar{v}_{e}$ flux is due to very short baseline, that is high $\Delta m_{j i}^{2}$, neutrino oscillations. (There are, however, many criticisms of this interpretation. For example, see [16].) Another example has to do with short baseline accelerator experiments [17].

All examples taken together suggest a consistent picture, with a best fit given by $\sin ^{2} 2 \theta \approx 0.1$ and $\Delta m^{2} \approx 1.8 \mathrm{eV}^{2}$, for one additional generation of neutrino [18]. However, the landscape is in principle wide open for scenarios with more than one extra generation. In this case, any number of new oscillation phenomena might be present.

Daya Bay recently published [13] a search for a sterile neutrino signal. Spectra were normalized, as shown in the left in Figure 4, relative to that observed in Experimental Hall 1 (EH1; see Figure 1) located near one of the two nuclear reactor locations. This would allow us to see distortions in the spectra for EH2 (the other near hall) and EH3 (the far hall) for the the range $10^{-3}<\Delta m^{2}<0.3 \mathrm{eV}^{2}$. Figure 4 shows also shows the expected magnitude of the distortion for two different values of $\Delta m^{2}$. Clearly our data is consistent with no large effects from sterile neutrinos in this mass range. The right panel shows our excluded region. Although we cannot exclude the best fit global value for one additional sterile neutrino [18], this result does constrain other models. 

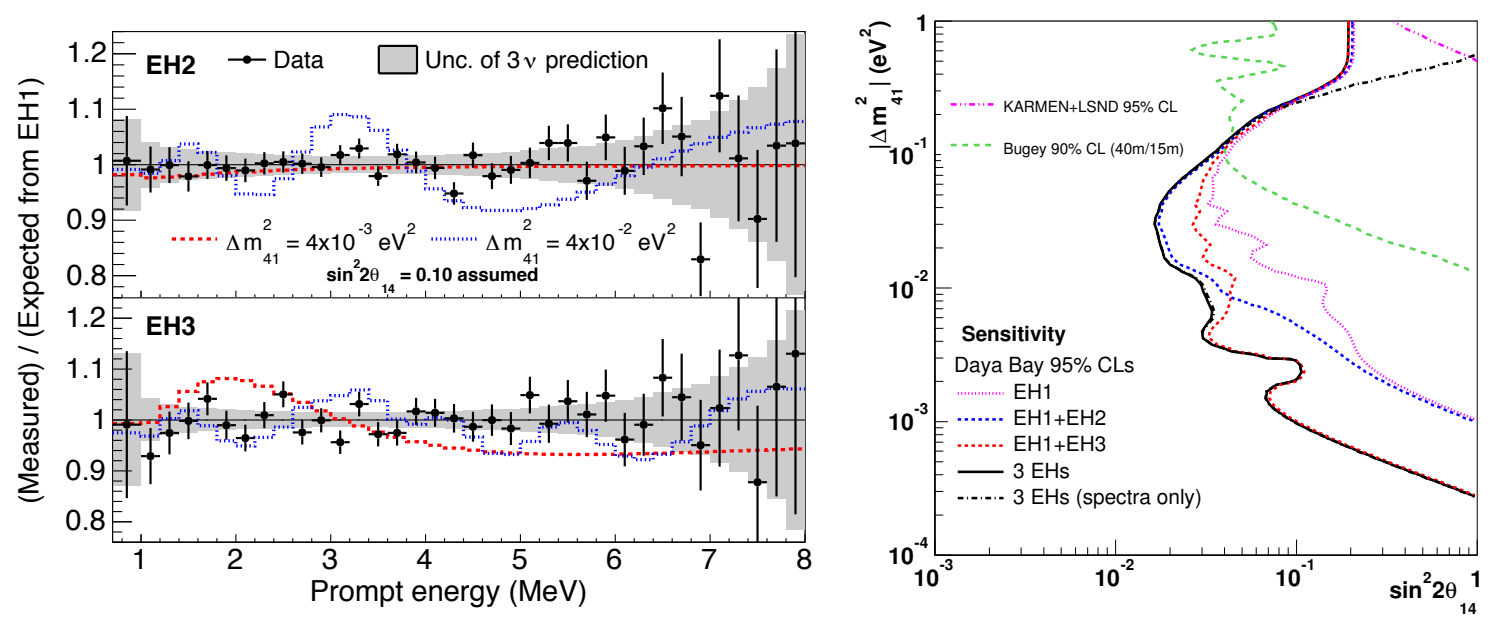

Figure 4: Spectral ratios and expected distortion from two different values of $\Delta m^{2}$, corresponding to a fourth "sterile" neutrino, along with our excluded region in this parameter and mixing angle. Figures taken from our recent publication [13] based on our first 217 days of data.

\section{Preliminary results on reactor flux and spectrum}

Our collaboration is nearing completion of an analysis of the absolute neutrino flux and spectrum shape. This necessitates tight control on systematic uncertainties associated with absolute energy and efficiency calibrations, and this talk also presented preliminary results based on the first 217 days of data, using six antineutrino detectors.

A first principles calculation of the reactor neutrino spectrum is quite difficult, especially for a commercial power plant reactor where the flux will change over time as the core evolves, producing more fissions from isotopes of plutonium over time. Historically, calculations are done using a technique that inverts measurements of beta spectra, taken under conditions that attempt to recreate the environment in a reactor core. These inversions are not unique, however and require additional assumptions that are, in some cases, difficult to quantify. For this work, we compare to two inversion calculations [19, 20] based on data from ILL [21] as well as an older first-principles derivation [22].

Figure 5 shows preliminary results from our analysis. We determine a neutrino yield $Y_{0}$ in units of $\mathrm{cm}^{2} / \mathrm{GW}$.day, or equivalently $\sigma_{f}$ in $\mathrm{cm}^{2} /$ fission, from our observed inverse beta decay rate, corrected for efficiencies and neutrino oscillations. The yield is calculated using calibrations for each of the six detectors in this data set. Each detector gives consistent results with each other after various corrections are applied. The common systematic error results in a value for $Y_{0}$ between 1.52 and $1.59 \times 10^{-18} \mathrm{~cm}^{2} / \mathrm{GW}$-day, generally lower than the predicted values. (This deficit is consistent with the reactor neutrino anomaly [15].)

Even though the general trend is to observe fewer neutrinos than predicted, the spectrum in Figure 5 also shows an enhancement near $5 \mathrm{MeV}$. This "bump" is rather surprising, but it has been seen in other recent high-statistics reactor neutrino experiments. A different approach [23] to calculating the reactor neutrino spectrum, however, seems to suggest that this enhancement is actually an inherent feature. Understanding this development thoroughly will require further 

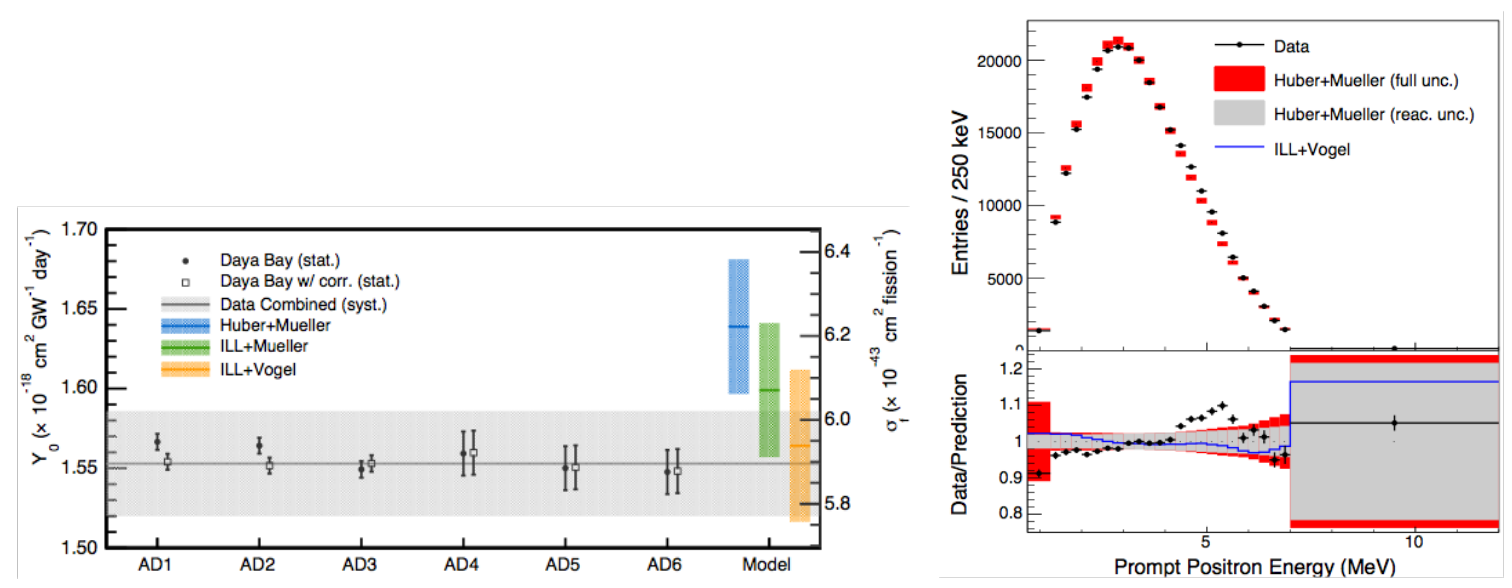

Figure 5: Preliminary results on the absolute neutrino flux and spectral shape from the Daya Bay collaboration. The left panel shows our flux determination with each of the six detectors from our initial data set. See the text for a discussion of the predicted values. The right plots the prompt energy spectrum compare to one calculation, showing the energy dependence of the overall deficit, but also an expected feature near $5 \mathrm{MeV}$.

analysis, new calculations, and quite possibly new experiments aiming more directly at measuring the fundamental neutrino flux.

\section{References}

[1] F. P. An et al. [Daya Bay Collaboration], "A side-by-side comparison of Daya Bay antineutrino detectors," Nucl. Instrum. Meth. A 685 (2012) 78 [arXiv:1202.6181 [physics.ins-det]].

[2] H. R. Band, R. Carr, X. C. Chen, X. H. Chen, J. J. Cherwinka, M. C. Chu, E. Draeger and D. A. Dwyer et al., "Assembly and Installation of the Daya Bay Antineutrino Detectors," JINST 8, T11006 (2013) [arXiv:1309.1557 [physics.ins-det]].

[3] W. Beriguete, J. Cao, Y. Ding, S. Hans, K. M. Heeger, L. Hu, A. Huang and K. B. Luk et al., "Production of a gadolinium-loaded liquid scintillator for the Daya Bay reactor neutrino experiment," Nucl. Instrum. Meth. A 763 (2014) 82 [arXiv:1402.6694 [physics.ins-det]].

[4] H. R. Band, J. J. Cherwinka, E. Draeger, K. M. Heeger, P. Hinrichs, C. A. Lewis, H. Mattison and M. C. McFarlane et al., "The Daya Bay Antineutrino Detector Filling System and Liquid Mass Measurement," JINST 8 (2013) P09015 [arXiv:1307.1089 [physics.ins-det]].

[5] H. R. Band, J. J. Cherwinka, M. C. Chu, K. M. Heeger, M. W. Kwok, K. Shih, T. Wise and Q. Xiao, “Daya Bay Antineutrino Detector Gas System," JINST 7 (2012) P11029 [arXiv:1210.0643 [physics.ins-det]].

[6] F. P. An et al. [Daya Bay Collaboration], "The Muon System of the Daya Bay Reactor Antineutrino Experiment," arXiv:1407.0275 [physics.ins-det].

[7] J. Wilhelmi, R. Bopp, R. Brown, J. Cherwinka, J. Cummings, E. Dale, M. Diwan and J. Goett et al., "The Water Purification System for the Daya Bay Reactor Neutrino Experiment," arXiv:1408.1302 [physics.ins-det].

[8] K. A. Olive et al. [Particle Data Group Collaboration], "Review of Particle Physics," Chin. Phys. C 38 (2014) 090001. 
[9] F. P. An et al. [Daya Bay Collaboration], "Observation of electron-antineutrino disappearance at Daya Bay,” Phys. Rev. Lett. 108 (2012) 171803 [arXiv:1203.1669 [hep-ex]].

[10] F. P. An et al. [Daya Bay Collaboration], "Improved Measurement of Electron Antineutrino Disappearance at Daya Bay,” Chin. Phys. C 37 (2013) 011001 [arXiv:1210.6327 [hep-ex]].

[11] F. P. An et al. [Daya Bay Collaboration], "Spectral measurement of electron antineutrino oscillation amplitude and frequency at Daya Bay,” Phys. Rev. Lett. 112 (2014) 061801 [arXiv:1310.6732 [hep-ex]].

[12] F. P. An et al. [Daya Bay Collaboration], "Independent Measurement of $\theta_{13}$ via Neutron Capture on Hydrogen at Daya Bay,” Phys. Rev. D 90 (2014) 071101 [arXiv:1406.6468 [hep-ex]].

[13] F. P. An et al. [Daya Bay Collaboration], "Search for a Light Sterile Neutrino at Daya Bay,” Phys. Rev. Lett. 113 (2014) 141802 [arXiv:1407.7259 [hep-ex]].

[14] M. Apollonio et al. [CHOOZ Collaboration], "Limits on neutrino oscillations from the $\mathrm{CHOOZ}$ experiment," Phys. Lett. B 466 (1999) 415

[15] G. Mention, M. Fechner, T. Lasserre, T. A. Mueller, D. Lhuillier, M. Cribier and A. Letourneau, "The Reactor Antineutrino Anomaly," Phys. Rev. D 83 (2011) 073006

[16] A. C. Hayes, J. L. Friar, G. T. Garvey, G. Jungman and G. Jonkmans, "Systematic Uncertainties in the Analysis of the Reactor Neutrino Anomaly,” Phys. Rev. Lett. 112 (2014) 202501

[17] J. M. Conrad, W. C. Louis and M. H. Shaevitz, "The LSND and MiniBooNE Oscillation Searches at High $\Delta m^{2}$, , Ann. Rev. Nucl. Part. Sci. 63 (2013) 45

[18] J. Kopp, P. A. N. Machado, M. Maltoni and T. Schwetz, "Sterile Neutrino Oscillations: The Global Picture," JHEP 1305 (2013) 050

[19] P. Huber, “On the determination of anti-neutrino spectra from nuclear reactors," Phys. Rev. C 84 (2011) 024617 [Erratum-ibid. C 85 (2012) 029901]

[20] T. A. Mueller, D. Lhuillier, M. Fallot, A. Letourneau, S. Cormon, M. Fechner, L. Giot and T. Lasserre et al., "Improved Predictions of Reactor Antineutrino Spectra," Phys. Rev. C 83 (2011) 054615

[21] K. Schreckenbach, G. Colvin, W. Gelletly and F. Von Feilitzsch, "Determination Of The Anti-neutrino Spectrum From U-235 Thermal Neutron Fission Products Up To 9.5-mev,” Phys. Lett. B 160 (1985) 325; F. Von Feilitzsch, A. A. Hahn and K. Schreckenbach, "Experimental Beta Spectra From Pu-239 And U-235 Thermal Neutron Fission Products And Their Correlated Anti-neutrinos Spectra,” Phys. Lett. B 118 (1982) 162; A. A. Hahn, K. Schreckenbach, G. Colvin, B. Krusche, W. Gelletly and F. Von Feilitzsch, "Anti-neutrino Spectra From ${ }^{241} \mathrm{Pu}$ and ${ }^{239} \mathrm{Pu}$ Thermal Neutron Fission Products,” Phys. Lett. B 218 (1989) 365.

[22] P. Vogel, G. K. Schenter, F. M. Mann and R. E. Schenter, "Reactor Anti-neutrino Spectra and Their Application to Anti-neutrino Induced Reactions. 2.,” Phys. Rev. C 24 (1981) 1543.

[23] D. A. Dwyer and T. J. Langford, "Spectral Structure of Electron Antineutrinos from Nuclear Reactors," arXiv:1407.1281 [nucl-ex]. 